\title{
tweeDe - A Universal Dependencies treebank for German tweets
}

\author{
Ines Rehbein \\ Leibniz ScienceCampus \\ Heidelberg University/ \\ IDS Mannheim \\ $\{$ rehbein|ruppenhofer\}@ids-mannheim.de
}

\author{
Bich-Ngoc Do \\ Leibniz ScienceCampus \\ Heidelberg University/ \\ IDS Mannheim \\ do@cl.uni-heidelberg.de
}

\begin{abstract}
We introduce the first German treebank for Twitter microtext, annotated within the framework of Universal Dependencies. The new treebank includes over 12,000 tokens from over 500 tweets, independently annotated by two human coders. In the paper, we describe the data selection and annotation process and present baseline parsing results for the new testsuite.
\end{abstract}

\section{Introduction}

Recent years have seen an increasing interest in developing robust NLP applications for data from different language varieties and domains. The Universal Dependencies (UD) project (Nivre et al., 2016) has inspired the creation of many new datasets for dependency parsing in a multilingual setting. Treebanks have been created for low-resourced languages such as Bambara, Erzya, or Kurmanji as well as for many new domains, genres and language varieties for which no annotated data was yet available. A case in point are web genres, spoken discourse, literary prose, historical data or data from social media. ${ }^{1}$

We contribute to the creation of new resources for different language varieties and introduce tweeDe, a new German UD Twitter treebank. TweeDe has a size of over 12,000 tokens, annotated with PoS, morphological features and syntactic dependencies. TweeDe is different from existing German UD treebanks as its content focusses on private communication. Private tweets share many properties of spoken language. They are often highly informal and not carefully edited, often lack punctuation and can include ungrammatical structures. In addition, the data often includes spelling errors and a creative use of language that results in a high number of unknown words. These properties make user-generated microtext a challenging test case for parser evaluation.

In the paper, we describe the creation of tweeDe, including data selection, preprocessing and the annotation process. We report inter-annotator agreement for the syntactic annotations ( $\$ 2)$ and discuss some of the decisions that we have made during annotation ( $\$ 3)$. We compare tweeDe to other treebanks in $\S 4$. In $\S 5$ we present baseline parsing results for the new treebank. Finally, we put our work into context (§6) and outline avenues for future work (\$7).

\section{2 tweeDe - A German Twitter treebank}

This section describes the creation of the first German Twitter treebank, annotated with Universal Dependencies. The treebank includes 519 tweets with over 12,000 tokens of microtext.

\subsection{Data extraction}

The annotation of user-generated microtext is a challenging task, due to the brevity of the messages and the missing context information, which often results in highly ambiguous texts. As a result, interannotator agreement (IAA) is often below the one obtained on standard newspaper text. To avoid such problems, we opted to extract short communication threads, which range in length from 2 up to 34 tweets. This approach allowed the annotators to see the context of each tweet and was thus crucial for resolving ambiguities in the data.

\footnotetext{
${ }^{1}$ The different treebanks and their description are available from: https://universaldependencies.org/.
} 
The conversations were collected in two steps. We first used an existing python tool $^{2}$ that supports the downloading of conversations by querying the Twitter API for a set of query terms and then scraping the $\mathrm{html}$ page on twitter.com that represents each matching conversation. However, Twitter does not embed complete json files into the html-pages and the existing crawler had some problems in fully retrieving tweet text containing certain special characters. We therefore used the output of the initial crawler only to establish the ids and the sequencing of the tweets in a conversation and then re-downloaded the full json files to be sure we had complete tweets.

The query terms we used were all German stop words, i.e. highly-frequent closed-class function words such as prepositions, articles, modal verbs, and adverbs such as auch 'too' or dann 'then'. The idea behind this was to avoid any kind of topic bias. Of the threads retrieved, we only retained those representing private communication between two or more participants. Threads consisting mainly of automatically generated tweets, advertisements, and so on were discarded after manual inspection. The treebank preserves the temporal order of the tweets in the same thread. For meta-information, we keep the tweet id, date and time as well as the author's user name. As is common practise for UD treebanks, we also store the raw, untokenised text for each tweet.

Besides issues arising from brevity, further problems for annotating user-generated social media content are the creative use of language, including acronyms (example 1) and emoticons (example 2), noncanonical spellings (example 3), missing arguments (example 2) and the often missing or inconsistent use of punctuaction (examples 1-4). The latter causes segmentation problems like those faced in annotating spoken language where, since no punctuation is given, the annotator has to decide on where to insert sentence boundaries.

(1) hdl have you dear "Love you"

(2)

Mache deshalb gerne mal mit $<3$
participate thus gladly MODAL PTCL VERB PTCL EMOTICON
"Hence (I) like to participate once in a while $<3$ "

(3) Is nich wahr ich habe nur einen report bekommen das sie es erhalten haben und überprüfen. is not true I have only a report got that they it received have and check.. "It's not true. I only got a report that they have received it and will check it."

(4) Mahlzeit Arbeit Gassigang Wohnung geputzt Essen gemacht Jaaaa es ist \#Freitag und jetzt meal work walking the dog flat cleaned food made Yeeees it is Friday and now \#hochdiehaendewochenende \#up-the-hands-weekend

\subsection{Segmentation}

For spoken German, several proposals have been made how to segment transcribed utterances, based on syntax, intonation and prosodic cues, pausing and hesitation markers (Rehbein et al., 2004; Selting et al., 2009). However, when the different levels of analysis provide contradicting evidence, it is not clear how to proceed. For tweets, we have to deal with similar issues. When no (or only inconsistent use of) punctuation is present, we have to decide how to segment the tweet into units for syntactic analysis. Earlier work has chosen to consider the whole tweet as one unit, i.e. as one syntax tree. Since Twitter has changed their policy and doubled the length limit from 140 to 280 characters, this is no longer feasible (see example 5 below). We thus decided to split up the messages into sentences, based on the following rules.

(5)@surfguard @Mathias59351078@ArioMirzaie Über einige amüsiere ich mich köstlich, bei manchen denke ich "hm" und bei wieder anderen bin ich entsetzt. Mit keinem einzigen hab ich irgendwas zu tun. Wenn du mich wegen meiner Hautfarbe den Schuldigen zuordnest, bist du ein Rassist.

“@surfguard @Mathias59351078 @ArioMirzaie Some make me laugh, some make me think "hm“ and still others make me feel appalled. I don't have anything to do with any of them. If you blame me for the color of my skin, you're a racist."

- Hashtags and URLs at the beginning or end of the tweet that are not syntactically integrated in the sentence are separated and form their own unit (tree).

- Emoticons are treated as non-verbal comments to the text and are integrated in the tree (figure 1).

\footnotetext{
${ }^{2}$ https://github.com/song9446/twitter-corpus-crawler-python
} 


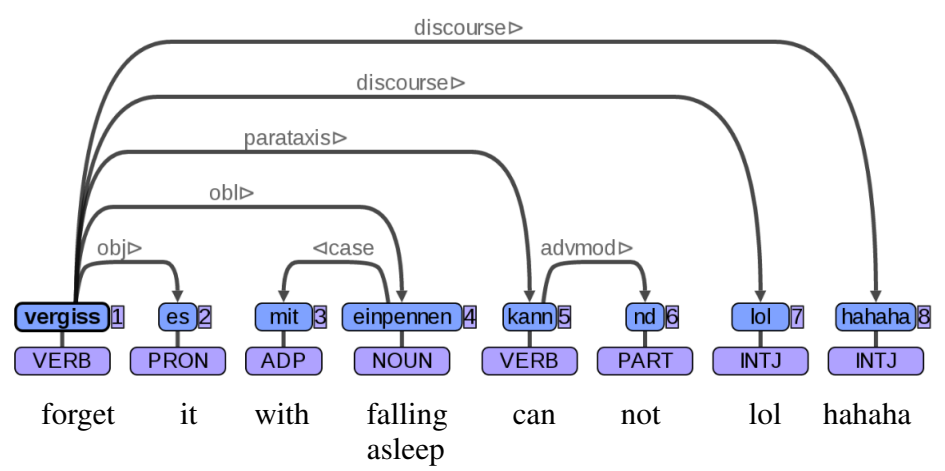

Figure 1: Example tree from tweeDe, displayed in UD-annotatrix (Tyers et al., 2018).

- Interjections (Aaahh), inflectives $\left(* g r i n s^{*}\right)$, fillers $(\ddot{a h m})$ and acronyms typical for social media content $(l o l, O M G)$ are also not separated but considered to be part of the tree (figure 1).

\subsection{Tokenisation}

User-generated text often reflects (or mimics) morpho-phonological processes from spoken language that are in conflict with the rules of Standard German orthography. One example are words merged into one token that, according to German grammar, should be separated but in spoken varieties of German are contracted into one token. We split merged tokens to avoid having tokens with more than one PoS tag and grammatical function. To mark that the word has been written as one atomic token, we use the UD feature SpaceAfter=No in combination with CorrectSpaceAfter=Yes in the last column of the CoNLL-UD file. Figure 2 (left) shows an example where the canonical token sequence "Kennst Du ?" is instead fused into the single token "Kennste?".

We also observe the opposite case where tokens that should have been written as one word are split into two or more separate tokens in the tweet. Most of these are German noun compounds. We chose to annotate split compounds using the UD relation goeswith. We follow UD conventions to always annotate the first component as the head and attach all remaining components to the first component. One problem with this approach is that in some cases the head of the compound will end up with the wrong PoS tag. Figure 2 (right) gives an example where the whole compound should have been annotated as a noun (Japanurlaub, Japan vacation) but instead now obtains a proper noun PoS tag. A possible solution to this problem is to deviate from UD practise and annotate the second component (i.e. the real head) as the head. As those cases were rare in our data, we refrained from doing so, for the sake of consistency with other UD treebanks.

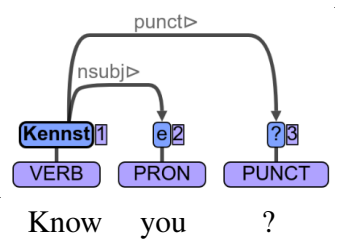

(6) Kennste ? (raw)

Kennst e ? (tokenised)

kennen du? (lemmatised)

"Do you know that?"

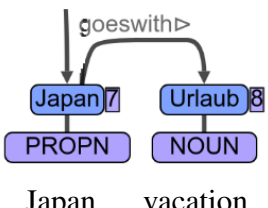

Japan vacation
(7) Japan Urlaub (raw) Japan Urlaub (tokenised) Japan Urlaub (lemmatised)

"vacation in Japan"

Figure 2: Merged tokens (left) and split compound (right)

\subsection{Annotation}

We annotated two types of PoS tags, based on the UD (Petrov et al., 2012) and Stuttgart-Tübingen (STTS) (Schiller et al., 1995) tag sets. The PoS tags and morphological features represent the annotations of one annotator, correcting the output of the UD processing pipeline for German (UDPipe) (Straka and Straková, 2017). For all dependency annotations, two annotators provided syntactic attachments and dependency labels, which were subsequently adjudicated. The adjudicated syntactic dependency relations were used for consistency checks between the dependency labels and the PoS and morphological tags. Additional consistency checks based on DECCA (Dickinson and Meurers, 2003) verified the compatibility of the different annotation layers. All incompatibilities were manually inspected and resolved. The final testsuite includes 12,073 tokens from 519 tweets, split up into train, development and test data (table 1). Around $10 \%$ of the tweets include a non-projective tree structure. 


\begin{tabular}{|l|r|c|r|rr|c|}
\hline tweeDe & \# tweets & \# tok & \# vocab & OOV & lower & \# non-projective \\
\hline train & 250 & 5,747 & 2,035 & 0 & 0 & 25 \\
dev & 69 & 1,917 & 861 & 520 & 479 & 6 \\
test & 200 & 4,409 & 1,661 & 1,157 & 1,034 & 21 \\
\hline total & $\mathbf{5 1 9}$ & $\mathbf{1 2 , 0 7 3}$ & $\mathbf{3 , 6 3 9}$ & - & - & $\mathbf{5 2}$ \\
\hline
\end{tabular}

Table 1: Corpus statistics for the tweeDe testsuite (OOV: number of out-of-vocabulary words with regard to the training set; lower: OOV for lower-cased word forms).

Inter-Annotator Agreement We computed IAA on a subset of the data with 1,630 tokens. For labelled attachments, the agreement between the two annotators was $0.83 \kappa$, for unlabelled attachments the score increased to $0.89 \kappa$.

\section{Annotation decisions}

Below we discuss decisions we made during the annotation process that deviate from other existing German UD treebanks, i.e. the UD-GSD and the UD-TüBa-D/Z. UD-GSD has been converted from an earlier version of Stanford-style dependencies (McDonald et al., 2013) and contains mostly web reviews while the UD-TüBa-D/Z (Çöltekin et al., 2017) is a conversion of the TüBa-D/Z (Telljohann et al., 2004) and includes articles from a German daily newspaper.

Placeholder sentences In the UD-GSD treebank, finite subordinate placeholder sentences with dass or $o b$ (that, whether) are mostly analysed as ccomp while infinite correlates are annotated as acl and attached to the placeholder, usually a pronominal adverb. In contrast, the TüBa-D/Z attaches both finite and infinite placeholder clauses as adverbial clause to the verb of the matrix clause.

We decided to annotate finite and infinite placeholder sentences as acl and attach both to their respective placeholder (figure 3).

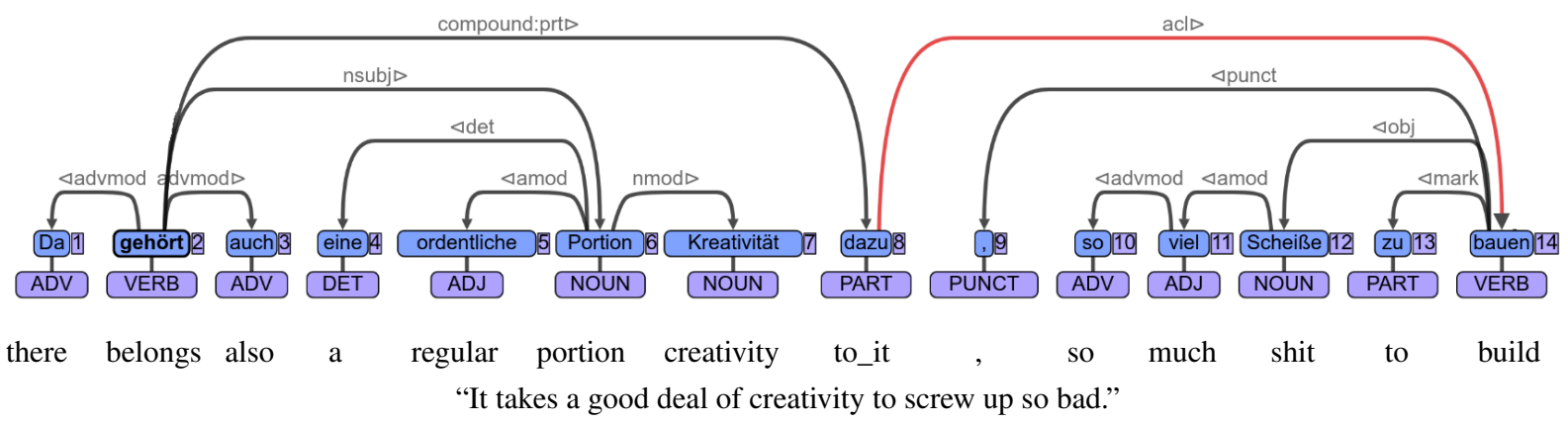

Figure 3: Placeholder sentence with pronominal placeholder.

Fixed multi-word constructions German has a rich system of adverbs and particles that can form multi-word constructions and so obtain a meaning that is different from the one of their individual components. We annotate those using the dependency label $\mathrm{f}$ ixed (figure 4 left). Adpositions also frequently form multiword units and have been treated the same (figure 4 right), as have specific combinations of pronouns and prepositions (e.g. Was für ein Unsinn! (What for a nonsense), English translation: "What utter nonsense!").
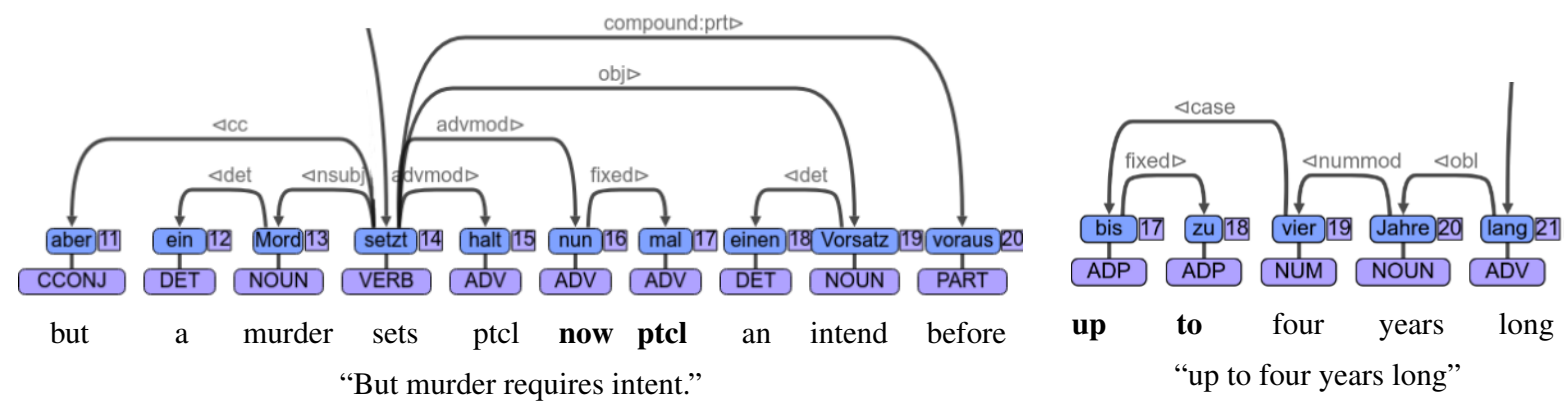

Figure 4: Particle multiword constructions (left) and adpositional multiwords (right). 


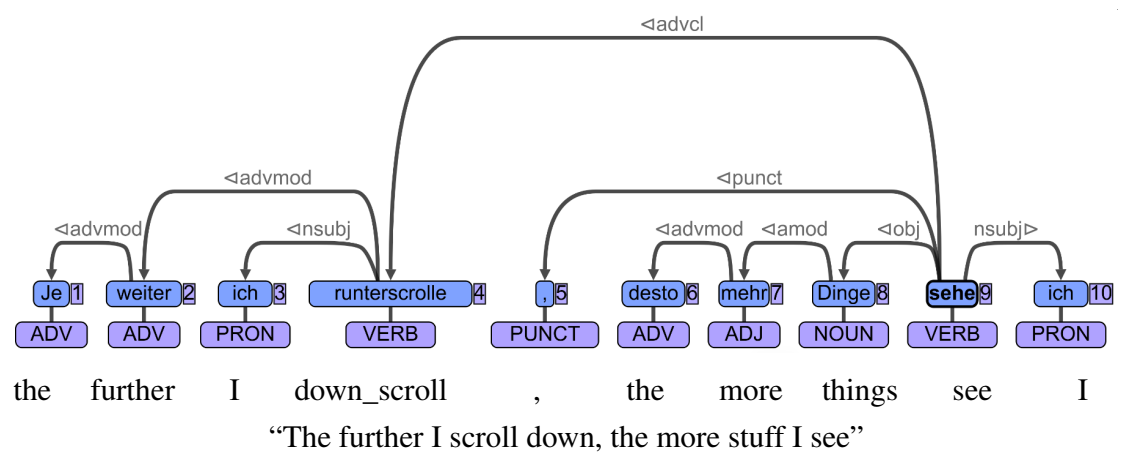

Figure 5: Comparative clause with je-desto in tweeDe.

Correlative construction with two clauses The correlative construction je $X$, desto/umso $Y$ (the $\mathrm{X}$, the $\mathrm{Y}$ ) (figure 5) consists of a subordinate clause marked by je, followed by a matrix clause that is introduced by desto/umso. ${ }^{3}$ Each clause needs to contain a comparative form, either of an adjective or of an adverb. Semantically, the construction describes a relationship between an independent and a dependent variable (example 8).

As indicated by word order, the clause expressing the causal variable is the subordinate clause (the finite verb comes last) while the clause describing the dependent variable is syntactically encoded as the matrix clause (the finite verb comes in second position). While je typically only marks the subordinate clause, there also exist variants of the construction where the desto/umso is omitted and a second je is used instead to mark the comparative that describes the dependent variable (example 9). ${ }^{4}$

(8) Je älter ich werde, umso glücklicher bin ich. PTCL older I become, PTCL happier am I.

"The older I get, the happier I am."
(9) Je größer die Gruppe, je kleiner der Preis. PTCL bigger the group, PTCL smaller the price. "The larger the group, the smaller the price."

Based on these observations, we decided to attach the subordinate clause as an adverbial clause to the matrix clause and analyse both particles as adverbial modifiers. We do not assign the mark relation as the particles are not modifiers of the head of the subordinate clause but are modifiers of the comparative forms in the subordinate and in the matrix clause.

This analyis is different from the one in the German UD-GSD and TüBa-D/Z UD treebanks (figure 6) where the head of the subordinate clause is analysed as the root of the sentence and the matrix clause is attached as a conjunct of the subordinate clause. Our analysis is consistent with the one for conditional clauses that are similar in meaning (e.g.: If I scroll down further, I can see more), where the subordinate if-clause is also an adverbial clausal modifier of the matrix clause.

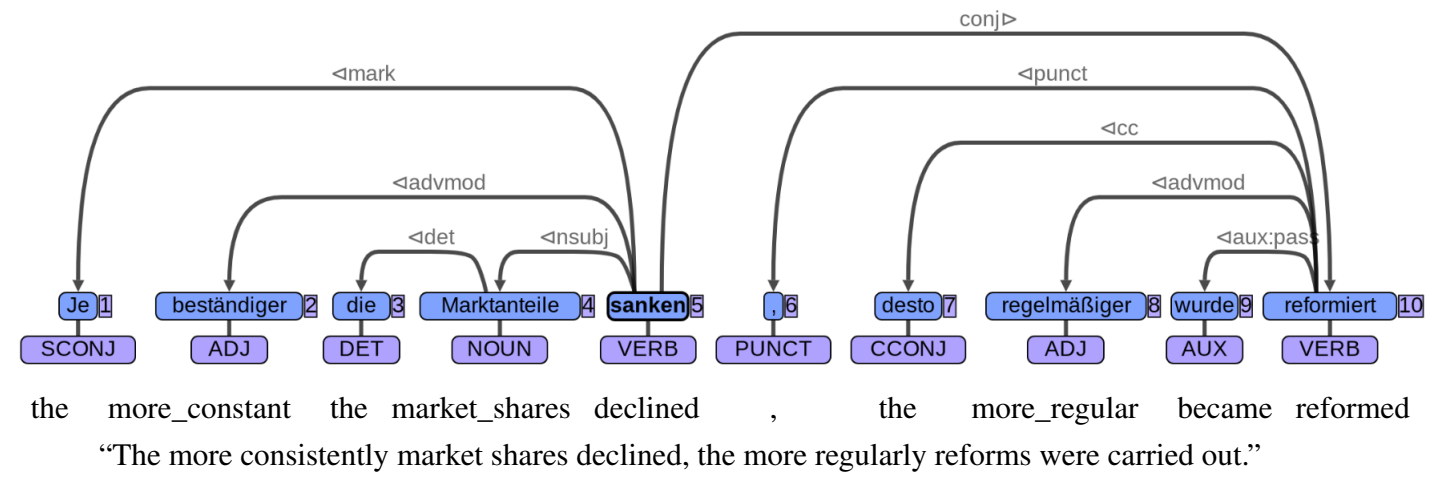

Figure 6: Comparative clause with je-desto in the TüBa-D/Z-UD.

\section{Comparison to other German UD treebanks}

We now compare tweeDe to three other German treebanks, i) UD-GSD, ii) TüBa-D/Z and iii) UD-HDT. The UD-HDT (Hennig and Köhn, 2017) is a conversion of the Hamburg Dependency Treebank (Foth et

\footnotetext{
${ }^{3}$ While this is the canonical order, it is also possible to switch the order of the matrix and subordinate clauses. Constructions without verbal predicates are also possible: Je mehr, desto lustiger. (The more, the merrier).

${ }^{4}$ While these are less frequent than the canonical form with je-desto/umso, it is easy to find instances in a large corpus such as the DeWac (Baroni et al., 2009), as well as instances that include only the je without a second particle where the matrix clause then needs to be in V1 word order.
} 


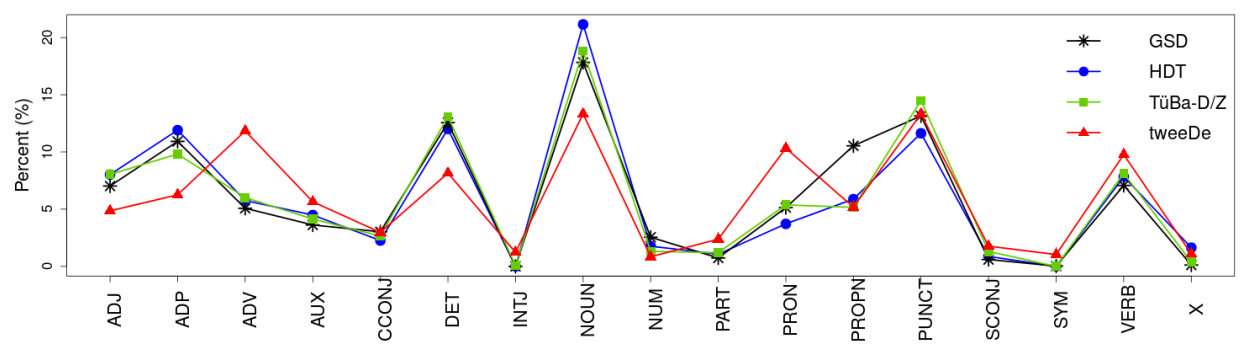

Figure 7: Distribution of UD PoS tags in four German UD treebanks.

al., 2014) which includes mostly news articles and is also the largest existing German treebank.

Figure 7 shows the distribution of PoS tags in the four treebanks. While the other three treebanks are quite homogeneous (except UD-GSD including more proper names), the most striking difference between tweeDe and the other treebanks is the higher number of adverbs and pronouns. This is typical for informal multiparty communication and is accompanied by a lower percentage of nouns, determiners, adjectives and adpositions as well as a slightly higher amount of verbs. This shows that tweeDe has a more verbal style, as opposed to the nominal style of the other treebanks.

\section{Parsing experiments}

We present parsing baselines for the new German UD treebank, using the state-of-the-art parser of Dozat et al. (2017). The parser is a neural dependency parser that learns complex, non-linear representations directly from the input text, based on bidirectional LSTMs (Hochreiter and Schmidhuber, 1997). It only considers local context and predicts attachments and labels in a greedy fashion. The huge success of the parser is based on its use of biaffine attention.

In our first experiment, we train the parser on the 250 tweets in the tweeDe training set. We use pretrained skipgram embeddings with 100 dimensions (window size: 5, min word count: 10), trained on a large collection of German tweets, collected in a time period from 2013 to 2017. The embeddings are publically available from https://www.cl.uni-heidelberg.de/research/downloads. All models have been trained with default parameters.

Table 2 (left) shows results for gold PoS and for automatically predicted PoS tags. Using UD PoS tags for parsing outperforms the STTS tags by a large margin, probably due to sparsity caused by the more fine-grained STTS. Feeding both, UD and STTS tags, to the parser can further increase results, but only slightly (less than 1\%). Most surprisingly, we obtain higher results when using automatically predicted STTS tags (as compared to using gold STTS tags). This observation, however, is more pronounced for the test set and might not be representative, being an artefact of the small data size.

Results for training on the small tweeDe dataset only are in the range of 74\% LAS (gold PoS) and 68\% LAS (auto PoS). When adding the training data from the German-GSD UD treebank, results increase to 81\% LAS (gold PoS) and 76\% LAS (auto PoS). The large gap of 5\% between the gold and auto PoS setting highlights the importance of high-quality PoS tags for parsing tweets.

\begin{tabular}{|l|l|l|l|l|l|}
\hline \multirow{5}{*}{ gold } & PoS & \multicolumn{2}{|c|}{ dev } & \multicolumn{2}{c|}{ test } \\
& tagset & UAS & LAS & UAS & LAS \\
\hline \multirow{4}{*}{ auto } & UD & 82.15 & 74.26 & 80.65 & 72.69 \\
& STTS & 73.48 & 63.05 & 70.28 & 60.83 \\
& BOTH & 82.51 & 74.94 & 81.51 & 74.34 \\
\hline & UD & 78.88 & 69.90 & 76.01 & 67.08 \\
& STTS & 72.91 & 63.21 & 71.25 & 62.64 \\
& BOTH & 79.09 & 70.73 & 76.60 & 68.14 \\
\hline
\end{tabular}

\begin{tabular}{|l|l|l|l|l|l|}
\hline & PoS & \multicolumn{2}{|c|}{ dev } & \multicolumn{2}{c|}{ test } \\
& tagset & UAS & LAS & UAS & LAS \\
\hline \multirow{3}{*}{ gold } & UD & 88.17 & 81.73 & 86.40 & 80.47 \\
& STTS & 85.21 & 77.32 & 81.38 & 74.02 \\
& BOTH & 88.89 & 82.67 & 87.15 & 81.01 \\
\hline \multirow{4}{*}{ auto } & UD & 85.88 & 78.20 & 82.91 & 76.03 \\
& STTS & 84.90 & 76.44 & 82.32 & 74.79 \\
& BOTH & 86.30 & 78.15 & 83.31 & 76.39 \\
\hline
\end{tabular}

Table 2: Parsing results for the Dozat parser on tweeDe, without (left) and with additional training data from the German-GSD UD treebank (right). 


\begin{tabular}{|l|r|r|r|r|}
\hline & \# token & \# tweets & LAS & (parser) \\
\hline EN (Foster et al. 2011) & n.a. & $519 *$ & 67.3 & Malt2006 \\
EN (Kong et al. 2014) & 12,149 & 840 & - & \\
EN (Liu et al. 2018) & 55,607 & 3,550 & 77.7 & D\&M2017 \\
EN-AAE (Blodgett et al. 2018) & 3,072 & 250 & 56.1 & D\&M2017 \\
EN-MS (Blodgett et al. 2018) & 3,524 & 250 & 67.7 & D\&M2017 \\
IT (Sanguinetti et al. 2018) & 124,410 & 6,712 & 81.5 & D\&M2017 \\
\hline
\end{tabular}

Table 3: Statistics for manually annotated treebanks (*Foster et al. only report \# sentences, not \# tweets. We expect the no. of tweets to be slightly lower than 500). The data of Blodgett et al. includes AAE and main-stream (MS) English tweets. The last two columns report results for the Dozat \& Manning parser (Dozat et al., 2017) (w/o domain adaptation) or the Malt parser from the literature.

\section{Related work}

Twitter treebanks exist not only for English (Kong et al., 2014; Liu et al., 2018; Blodgett et al., 2018) but also for Italian (Sanguinetti et al., 2018) and Arabic (Albogamy et al., 2017). Foster et al. (2011) were among the first to provide syntactic analyses for Twitter microtext. They created a testset with over 500 sentences extracted from tweets. The data was automatically parsed with a constituency parser and the trees were manually corrected by one annotator. Inter-annotator agreement (IAA) for labelled bracketing, measured on a subset of the data annotated by a second annotator, was quite high with nearly 96\%. Parsing accuracy without any domain adaptation, however, was low: the Malt parser (Nivre et al., 2006), trained on the WSJ, achieved an LAS of $63.3 \%$ on the Twitter testset.

The Tweebank v1 (Kong et al., 2014) is another English Twitter treebank, with a size of over 900 tweets annotated with unlabelled dependencies. Liu et al. (2018) extend the work of Kong et al. (2014) by enlarging the treebank to more than 3,500 tweets, refining the guidelines and adding labels to the former unlabelled trees. They report an IAA of $84.3 \%$ for labelled attachments in the Tweebank v2. A third English Twitter treebank was created by Blodgett et al. (2018). Their corpus includes 250 AfricanAmerican English (AAE) tweets and 250 tweets of mainstream American English microtext. The data has been annotated by two coders but no inter-annotator agreement is reported.

The Italian Twitter treebank of Sanguinetti et al. (2018) is the largest existing Twitter treebank and includes more than 6,700 trees. The authors report an IAA of $0.92 \kappa$ for syntactic annotation. The results for a dependency parser (Dozat et al., 2017) trained on a combination of the Italian UD treebank and the new dataset are also quite high, with a labelled attachment score of $81.5 \%$. The high agreement and parsing scores suggest that the dataset is somewhat easier and more well-behaved than the Tweebank (see table 3 for baseline results for the different Twitter treebanks).

For Arabic, a treebank with Twitter microtext has been created fully automatically, based on predictions of a rule-based and a data-driven parser (Albogamy et al., 2017). Efforts have been made to map the annotations to the UD scheme, but, to the best of our knowledge, the data is not yet available.

With over 12,000 tokens, our new German Twitter treebank is comparable in size to TwEEBANK v1 (Kong et al., 2014) even though the number of tweets in our dataset is smaller. This is due to the fact that our data were collected after Twitter raised the maximum length for tweets from 140 to 280 characters.

\section{Conclusions}

We presented tweeDe, the first German Twitter treebank, as a new training and testsuite for UD parsing. TweeDe includes more than 12,000 tokens of informal private communication, annotated for PoS, morphology and UD syntactic dependencies. The data will be made available to the research community. ${ }^{5}$

We also presented parsing baselines for the new dataset, showing that combining a small amount of in-domain Twitter data in combination with a larger amount of out-of-domain data can yield parsing accuracies in the range of $83 \%$ (UAS) and $76 \%$ (LAS) on the new testsuite.

\footnotetext{
${ }^{5}$ https : //www. cl. uni-heidelberg.de/research/downloads.
} 


\section{References}

Fahad Albogamy, Allan Ramsay, and Hanady Ahmed. 2017. Arabic tweets treebanking and parsing: A bootstrapping approach. In The 3rd Arabic Natural Language Processing Workshop, WANLP 2017, pages 94-99.

Marco Baroni, Silvia Bernardini, Adriano Ferraresi, and Eros Zanchetta. 2009. The wacky wide web: a collection of very large linguistically processed web-crawled corpora. Language Resources and Evaluation, 43(3):209_ 226.

Su Lin Blodgett, Johnny Wei, and Brendan T. O’Connor. 2018. Twitter Universal Dependency Parsing for AfricanAmerican and Mainstream American English. In The 56th Annual Meeting of the Association for Computational Linguistics, ACL 2018, pages 1415-1425, Melbourne, Australia.

Çă̆rı Çöltekin, Ben Campbell, Erhard Hinrichs, and Heike Telljohann. 2017. Converting the TüBa-D/Z Treebank of German to Universal Dependencies. In The NoDaLiDa 2017 Workshop on Universal Dependencies, UDW 2017, pages $27-37$.

Markus Dickinson and W. Detmar Meurers. 2003. Detecting errors in part-of-speech annotation. In 10th Conference of the European Chapter of the Association for Computational Linguistics, EACL 2003, pages 107-114.

Timothy Dozat, Peng Qi, and Christopher D. Manning. 2017. Stanford's Graph-based Neural Dependency Parser at the CoNLL 2017 Shared Task. In Proceedings of the CoNLL 2017 Shared Task: Multilingual Parsing from Raw Text to Universal Dependencies, pages 20-30.

Jennifer Foster, Özlem Çetinoglu, Joachim Wagner, Joseph Le Roux, Stephen Hogan, Joakim Nivre, Deirdre Hogan, and Josef van Genabith. 2011. \#hardtoparse: POS tagging and parsing the twitterverse. In Analyzing Microtext, Papers from the 2011 AAAI Workshop, San Francisco, California, USA, August 8, 2011.

Kilian A. Foth, Arne Köhn, Niels Beuck, and Wolfgang Menzel. 2014. Because size does matter: The Hamburg Dependency Treebank. In The 9th International Conference on Language Resources and Evaluation, LREC 2014, pages 2326-2333.

Felix Hennig and Arne Köhn. 2017. Dependency tree transformation with tree transducers. In The NoDaLiDa Workshop on Universal Dependencies, UDW@ NoDaLiDa 2017, pages 58-66.

Sepp Hochreiter and Jürgen Schmidhuber. 1997. Long Short-Term Memory. Neural Computation, 9(8):17351780.

Lingpeng Kong, Nathan Schneider, Swabha Swayamdipta, Archna Bhatia, Chris Dyer, and Noah A. Smith. 2014. A dependency parser for tweets. In The 2014 Conference on Empirical Methods in Natural Language Processing, EMNLP 2014, pages 1001-1012, Doha, Qatar.

Yijia Liu, Yi Zhu, Wanxiang Che, Bing Qin, Nathan Schneider, and Noah A. Smith. 2018. Parsing tweets into universal dependencies. In The 2018 Conference of the North American Chapter of the Association for Computational Linguistics: Human Language Technologies, NAACL-HLT 2018, pages 965-975, New Orleans, Louisiana, USA.

Ryan T. McDonald, Joakim Nivre, Yvonne Quirmbach-Brundage, Yoav Goldberg, Dipanjan Das, Kuzman Ganchev, Keith B. Hall, Slav Petrov, Hao Zhang, Oscar Täckström, Claudia Bedini, Núria Bertomeu Castelló, and Jungmee Lee. 2013. Universal dependency annotation for multilingual parsing. In The 51st Annual Meeting of the Association for Computational Linguistics, ACL 2013, pages 92-97.

Joakim Nivre, Johan Hall, and Jens Nilsson. 2006. Maltparser: A data-driven parser-generator for dependency parsing. In The 5th International Conference on Language Resources and Evaluation, LREC 2006, pages 2216-2219.

Joakim Nivre, Marie-Catherine de Marneffe, Filip Ginter, Yoav Goldberg, Jan Hajič, Christopher D. Manning, Ryan McDonald, Slav Petrov, Sampo Pyysalo, Natalia Silveira, Reut Tsarfaty, and Daniel Zeman. 2016. Universal dependencies v1: A multilingual treebank collection. In The Tenth International Conference on Language Resources and Evaluation, LREC 2016, pages 1659-1666.

Slav Petrov, Dipanjan Das, and Ryan McDonald. 2012. A universal part-of-speech tagset. In The 8th International Conference on Language Resources and Evaluation, LREC 2012, pages 2089-2096, May.

Jochen Rehbein, Thomas Schmidt, Bernd Meyer, Franziska Watzke, and Annette Herkenrath. 2004. Handbuch für das computergestützte Transkribieren nach HIAT. Sonderforschungsbereich 538. 
Manuela Sanguinetti, Cristina Bosco, Alberto Lavelli, Alessandro Mazzei, Oronzo Antonelli, and Fabio Tamburini. 2018. PoSTWITA-UD: an Italian Twitter Treebank in Universal Dependencies. In The 11th International Conference on Language Resources and Evaluation, LREC 2018, pages 1768-1775.

Anne Schiller, Simone Teufel, and Christine Thielen. 1995. Guidelines für das Tagging deutscher Textkorpora mit STTS. Technical report, Universität Stuttgart, Universität Tübingen.

Margret Selting, Peter Auer, Dagmar Barth-Weingarten, Jörg R. Bergmann, Pia Bergmann, Karin Birkner, Elizabeth Couper-Kuhlen, Arnulf Deppermann, Peter Gilles, Susanne Günthner, et al. 2009. Gesprächsanalytisches Transkriptionssystem 2 (GAT 2). Gesprächsforschung: Online-Zeitschrift zur verbalen Interaktion.

Milan Straka and Jana Straková. 2017. Tokenizing, POS Tagging, Lemmatizing and Parsing UD 2.0 with UDPipe. In Proceedings of the CoNLL 2017 Shared Task: Multilingual Parsing from Raw Text to Universal Dependencies, pages 88-99, Vancouver, Canada, August. Association for Computational Linguistics.

Heike Telljohann, Erhard W. Hinrichs, and Sandra Kübler. 2004. The Tüba-D/Z Treebank: Annotating German with a Context-Free Backbone. In The Fourth International Conference on Language Resources and Evaluation, LREC 2004.

Francis M. Tyers, Mariya Sheyanova, and Jonathan North Washington. 2018. Ud annotatrix: An annotation tool for universal dependencies. In The 16th International Workshop on Treebanks and Linguistic Theories, TLT 2016, pages 10-17. 